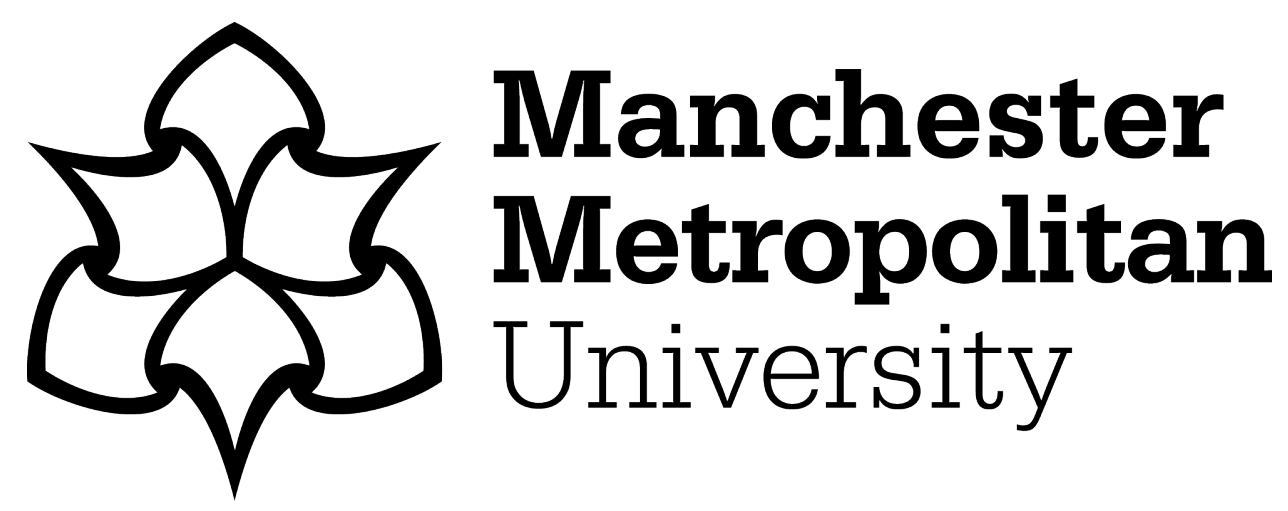

Bashir, AK ORCID logoORCID: https://orcid.org/0000-0001-7595-2522, Ohsita, Y and Murata, M (2016) Abstraction Layer Based Virtual Data Center Architecture for Network Function Chaining. In: 36th International Conference on Distributed Computing Systems Workshops, 27 June 2016 - 30 June 2016, Nara, Japan.

Downloaded from: https://e-space.mmu.ac.uk/624083/

Publisher: https://ieeexplore.ieee.org/abstract/document/7756205 DOI: https://doi.org/10.1109/ICDCSW.2016.42

Please cite the published version 


\title{
Abstraction Layer Based Virtual Data Center Architecture for Network Function Chaining
}

\author{
Ali Kashif Bashir, Yuichi Ohsita, and Masayuki Murata \\ Graduate School of Information Science and Technology \\ Osaka University \\ Osaka, Japan. \\ e-mail: \{ali-b, y-ohsita, murata\}@ist.osaka-u.ac.jp
}

\begin{abstract}
Network virtualization is one of the most promising technology for the data centers. It was innovated to use the network resources efficiently to evaluate new protocols and services on the same hardware. This paper discusses a virtual distributed data center network architecture in the network function virtualization environment. This architecture virtualize physical resources into multiple virtual clusters where each cluster consists of a group of machines (physical/virtual) and an abstraction layer. Optical technologies are used to construct the core of the network. Our abstraction layer construction algorithm selects the minimum optical switches that provide connectivity to all the machines of the group. One of the main use case of this architecture is orchestration of network function chains, where each chain corresponds to one cluster and network functions can be deployed over the optical switches of the abstraction layer. We also showed that this deployment can also save expensive optical/electronic/optical conversion cost.
\end{abstract}

Keywords-network virtualization; virtual data center network architecture; optical cloud; network function virtualization; network function chaining

\section{INTRODUCTION}

Data center networks (DCNs) are experiencing a rapid growth in both scale and complexity as they can host largescale applications and are acting as a backbone for clouds [1]. Companies like Amazon EC. [2], Microsoft Azure [3], Facebook [4], and Yahoo [5] routinely use data centers for storage, search, and computations. Such growth imposes a huge challenge to upgrade the current architectures of data centers. However, the current architecture is owned by a large number of Internet Service Providers and it is impossible to adopt new architectures without the agreement of all stakeholders.

Network Virtualization (NV) [6] [7] is one of the most promising technologies for the data centers (DCs). Introduced as a mean to evaluate new protocols and services [8]. It is already being actively used in research test-beds and applied in distributed cloud computing environments [9]. Now, it is seen as a tool to overcome the obstacles of the current internet to fundamental changes. As such, NV can be thought of as an inherent component of the future internet architecture [10]. For DCs, it works as a backbone technology and let concurrent applications execute on a single hardware. Today, NV approaches are even applied in the telecommunication market, e.g., Open-Flow [11].
With virtualization, we can create multiple logical Virtual Machines (VMs) on a single server to support multiple applications. However, virtualization of DCNs aims at creating multiple Virtual Networks (VNs) at the top of a single or multiple physical networks. VN, a primary entity in $\mathrm{NV}$, is a combination of active and passive network elements (nodes and links) lies on top of physical network/s. Virtual nodes are interconnected through virtual links, forming a virtual topology. With node and link virtualization, multiple VN topologies can be created and co-hosted on the same physical infrastructure. This virtualization introduces an abstraction that allows network operators to manage and modify networks in a highly flexible and dynamic way. On the other hand, without virtualization, we are limited to place a VM and also are limited in replacing or moving it.

The concept of Network Function Virtualization (NFV) was proposed within the European Telecommunication Standards Institute (ETSI) consortium [12] to provide innovation to the service delivery mechanism. NFV furnishes an environment where Network Functions (NFs) can be virtualized into Virtual Network Functions (VNFs). Currently, NFs are provided in terms of middle boxes, such as firewalls, Deep Packet Inspection (DPI), load balancers, etc. With virtualization and cloud technologies, NFV allows NFs, offered by specialized equipment, to run in software on generic hardware. Therefore, with NFV we can deploy VNFs when and where required. On the other hand, Network Function Chaining (NFC) [13] is a service deployment concept that exploits the features of the NFV and Software Defined Networking (SDN). An NFC consists of several NFs and VNFs.

In this paper, we discussed over architecture [14] [15] named Abstraction Layer based Virtual Clusters (AL-VC) in NFV environment. AL-VC was proposed to group VMs according to network service types, e.g. VMs offering Mapreduce services can be grouped together and VMs offering web services can be grouped separately, and so on. Note that, the number of services in a data center is defined by the network operator. An abstraction layer (AL) is the set of switches that used to manage the cluster. It selects the minimum set of switches that connect all the nodes. A particular group of VMs and its corresponding AL forms a Virtual Cluster (VC). This architecture offers several features to the underlying infrastructure, few of them were discussed in our previous works, such as low network update costs [14], flexibility and scalability [15]. In this work, we first 
proposed a new method for the construction of ALs that uses the vertex-cover algorithm to connect all the VMs. Moreover, we explained AL-VC in NFV environments. In NFV environments, NFCs are being orchestrated to meet the application demands. AL-VC provides a potential virtual architecture for the implementation of NFCs over it, where ALs can be used to implement the VNFs. Deployment of VNFs over optical domain can also save the optical/electronic/optical $(\mathrm{O} / \mathrm{E} / \mathrm{O})$ conversion cost.

The rest of the paper is organized as follows: in Section II, we discuss related works. In Section III, we present the overview of the architecture, and an algorithm for the construction of ALs. In Section IV, we explained the concept of NFCs and discussed AL-VC in the NFV environment for the orchestration of NFCs. Section V concludes the article.

\section{RELATED WORKS}

In this section, we will discuss the most relevant work on the virtualized of DCNs and NFV.

Virtualized DCNs can be categorized into centralized architecture and distributed architectures. First, we will talk about the main centralized architectures. MobileFlow [16] introduces carrier-grade virtualization in EPC. Diverter [17] is a software based network virtualization approach that does not configure switches or routers. It logically partition IP networks for better accommodations of applications and services. VL2 [18] is a data center network architecture that aims at achieving flexibility in resource allocation. Another VN architecture, CloudNaas [19] provides support for deploying and managing enterprise applications in the clouds. It relies on OpenFlow forwarding [11]. In NetLord [20], a tenant wanting to run a Map-Reduce task might simply need a set of VMs that can communicate via TCP. On the other hand, a tenant running a three-tier Web application might need three different IP subnets, to provide isolation between tiers. Or a tenant might want to move VMs or entire applications from its own datacenter to the cloud, without needing to change the network addresses of the VMs.

On the other hand, PolyVine [21] and adaptive VN [22] are two more worth discussing distributed approaches. Polyvine embeds end to end VNs in decentralized manners. Instead of technical, it resolves the legal issues among infrastructure providers. In adaptive VNs [22], every server is supposed to have an agent. Each server agent communicates with another to make local decisions. This approach is expensive and needs additional hardware. Apart from these, in [23], the authors surveyed on the importance of virtualization to improve flexibility, scalability, and resource utilization for data center networks. SecondNet [24] focused on providing bandwidth guarantees among VMs in a multi-tenant virtualized DC.

In NFV literature, Han et al. [25] presented the key technological requirements of the NFV; introduced NFV architectural framework and standardized activities. Moreover, they described some use cases of NFV, such as virtualization of mobile base station, home network, etc. Munoz et al. [26] discussed an architecture for SDN/NFV orchestration of SDN controller for multi-tenant optical networks. This architecture introduces SDN controller as a VNF and offer it in the cloud for dynamic use. Apart from these, some authors discussed the placement of service functions. For example, Sekar et al. [27] proposed to run software-centric middle-boxes on general-purpose hardware platforms with open application programing interfaces (APIs). Sherry et al. [28] proposed a method to deploy middle-boxes in the cloud.

\section{SYSTEM OVERVIEW}

\section{A. Motivation for Service based Clustering}

VCs are more desirable than physical DCs because the resource allocation to VCs can be rapidly adjusted as users' requirements change with time [24]. In DCNs, two machines (physical or virtual) providing similar service have high data correlation in comparison with servers providing different service [19]. In other words, in order to execute one service request, two machines offering identical services are likely to interact with each other more often than machines hosting different services. Logical representation of virtual clustering is shown in Figure 1, where a DCN is virtualized into multiple VCs of different service types.

One of the motivations for this sort of architecture is that the DCs usually store their data on servers according to data type, such as file servers, data servers, backup servers, etc. This kind of architecture can also help network operators to cluster their data center according to the different packages they offer to users. This kind of clustering offers scalability and flexibility to the devices and can save search and allocation time of queries.

\section{B. AL-VC topology}

Ideally, VN topology should be constructed in a way that it achieves minimum energy consumption and larger bandwidth without delay. The proposed architecture is capable of providing all these features. However, this is not the scope of this paper. Topology of AL-VC is presented in Figure 2, where all the servers in a server rack are connected to one Top-of- the-Rack (TOR) switch. Each server is

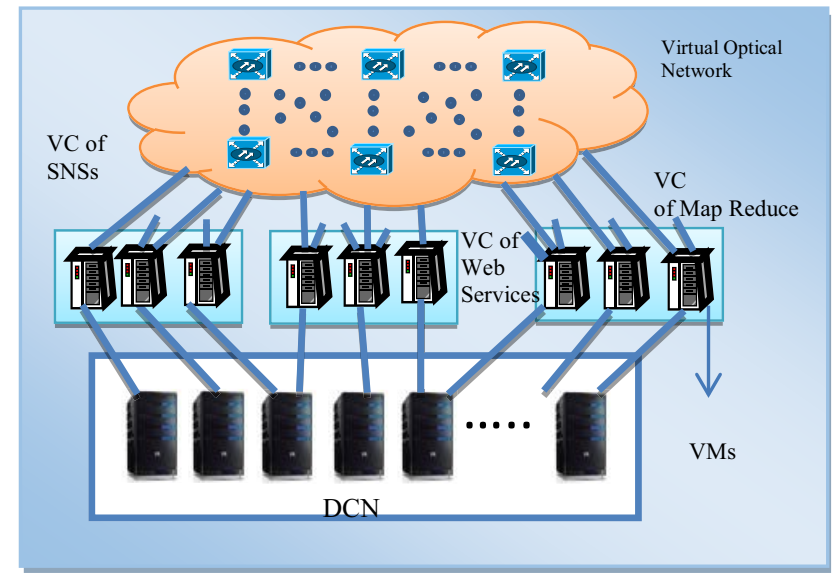

Figure 1. Overview of AL-VC 


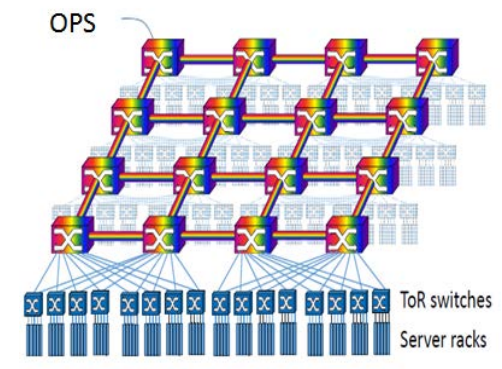

Figure 2. Topology of AL-VC

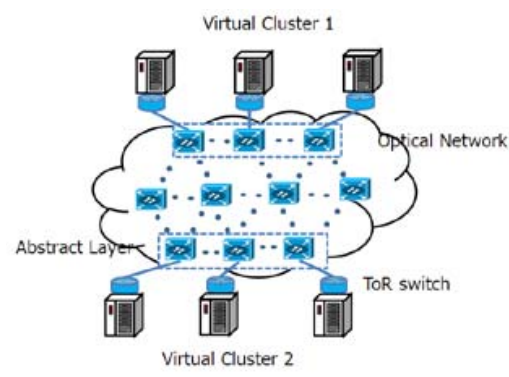

Figure 3. Virtual Clusters

hosting multiple VMs. In the core of the network, to construct the virtual topology, we use Optical Packet Switches (OPSs). Each TOR is connected to multiple OPSs. TOR switches produce electronic packets and they need to be converted into optical packets before sending over the optical domain. At the destination router, optical packets will be converted back to the electronic packets before forwarding to the TOR switches. This back and forth conversions results in $\mathrm{O} / \mathrm{E} / \mathrm{O}$ conversions that consumes an enormous amount of energy that should be reduced to increase the network performance.

Note that, the proposed topology can be constructed using electronic switches. However, in order to achieve higher bandwidth with small energy consumption, we use OPS [29].

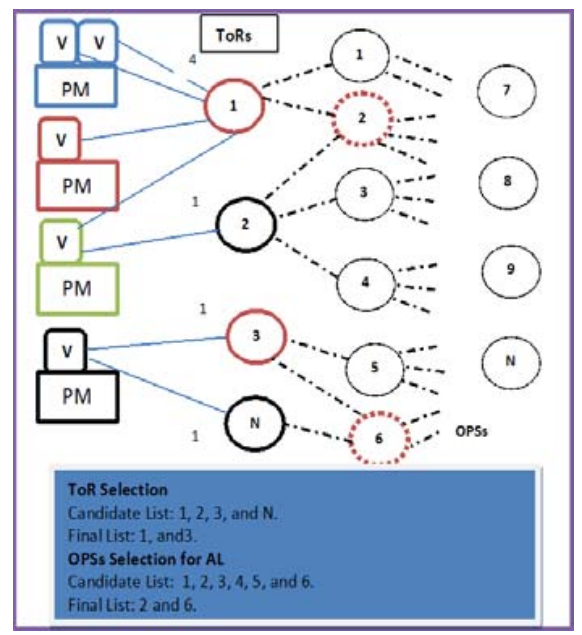

Figure 4. Construction of ALs

\section{Abstraction Layer}

$\mathrm{AL}$ is the key concept of this paper and is constructed by logically assigning a subset of the OPSs to a group of VMs that together is called a cluster in this paper. We assume that one OPS cannot be part of two ALs at the same time. The logical representation of AL is presented in Figure 3. An AL can be formed in several ways. In our previous works [15], we use random selection approach. In this work, we use the vertex cover and max-weightage algorithms to construct ALs. VMs of every cluster selects the minimum subset of OPSs that connects them as shown in Figure 4 where PM represents the physical machine and V the VM. We consider four ToR switches as $1,2,3$, and $\mathrm{N}$, where each TOR is connected to multiple OPSs. Objective is to select the minimum set of OPSs that connect all the VMs. Followings are the details steps of our algorithms.

- First, using the vertex cover algorithm, we draw a bipartite graph that connects all the VMs to ToRs and selects the minimum set of vertices. Consider a graph $G=(V, E)$, where $V$ represents the vertices and $E$ edges

$$
S \subseteq V \text { is a vertex cover if }
$$

$$
\forall\{v, \varpi\} \in E: v \in S \vee \varpi \in S
$$

minimum vertex cover (MIN-VCP): find a

vertex cover $\mathrm{S}$ that minimizes $|\mathrm{S}|$.

- Our algorithm then use the maximum-weighted algorithm to select the ToRs that cover all the VMs using maximum incoming and outgoing connections. By this, our algorithm selects first ToR 1 as it has four incoming connections and two outgoing. All the machines against the incoming connections will be considered covered and all the OPSs that connected to this switch will be taken into consideration. After this, it tries to select ToR 2 and notices that machines against this switch are already connected by ToR 1 . Next, it selects TOR 3 and notice that all the machines are being covered.

- Similarly, using the maximum-weighted algorithm, we select the OPSs against the selected ToRs as shown in the figure and this set of OPSs will be declared as the final AL as shown in the Figure 4.

- This procedure will be repeated for every group of VMs.

\section{AL-VC IN NFV ENVIRONMENTS}

In this section, we will first explain the concept of NFCs and then we will discuss that how AL-VC can be adopted in NFV environment for the orchestration of multiple NFCs. In the last section, we will explain that how deploying VNFs in the optical domain can save $\mathrm{O} / \mathrm{E} / \mathrm{O}$ conversions.

\section{A. NFCs}

One of the use cases for AL-VC is orchestration of NFCs in NFV. An NFC is a service deployment concept that exploits the features of NFV and SDN. An NFC is defined as a set of Network Functions (NFs), packet processing order (simple or complex), network resource requirements (node 


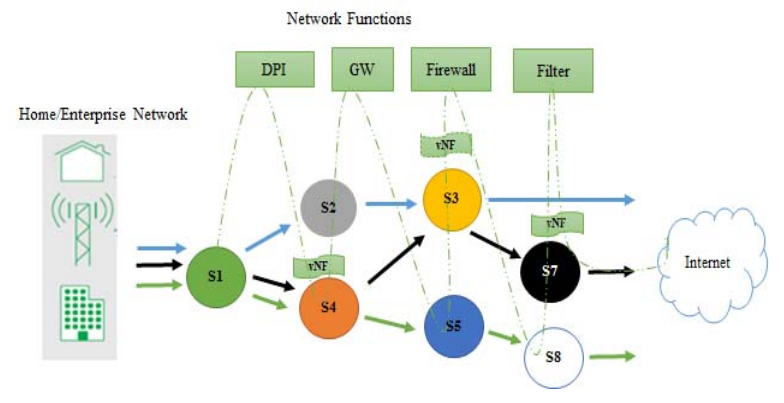

Figure 5. NFCs: The three arrows: blue, black, and green shows the pathline of three service chains, The dashed lines shows the functions (physical and virtual) on the NFCs

and links), and network forwarding graph. With NFCs, network operators can configure software dynamically without making any changes to the hardware. In this work, we consider the per-user/per-application service chaining. In the core of the network, we use optical technologies.

In Figure 5, three dynamic NFCs are given, where each NFC follows its own path. Nodes on the path are presented with $S_{1}, S_{2}, \ldots, S_{8}$ and each NFC orchestrates NFs/VNFs according to their demands. NFC can be implemented as a dynamic NFC where each flow processed by various NFs such as security gateways (GWs), firewalls, DPI, etc.

\section{B. AL-VC in NFV for NFCs}

In Figure 6, we presented the functional blocks of the NFV based AL-VC architecture. The physical network can consist of one or multiple DCNs that are constructed using the conventional TOR switches. On top of this, we deploy a virtualization layer responsible for virtualizing network resources. It abstracts the physical resources and anchors the VNFs to the virtualized infrastructure. Mainly, it is based on two NFVI managers, SDN controller and cloud/NFV manager. SDN controller provision, control, and manage the optical network and provide virtual connectivity services to users between VMs hosting VNFs. On the other hand,

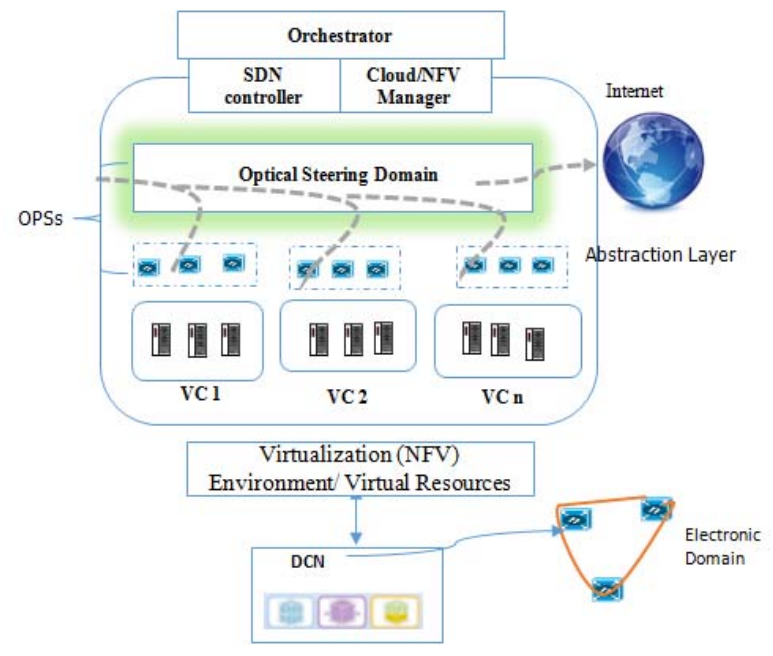

Figure 6. AL-VC in NFV
Cloud/NFV manager is responsible for managing VMs and storage resources. Moreover, it is also responsible for managing the VNFs during its lifetime, such as VNF creation, scaling, termination, and update events during the life cycle of VNF.

On top of this architecture, we proposed a network orchestrator for multiple-tenant SDN-enabled network. It is responsible for managing (provisioning, creation, modification, upgradation, and deletion) of multiple NFCs. It will logically divide the optical network into virtual slices and will allocate each slice to a single NFC. In AL-VC, that division is in the shape of ALs.

\section{NF and VNFs over $A L-V C$}

An NFC consists of a set of NFs or VNFs or both. In VNF environment, NFs when virtualized into VNFs can be deployed anywhere anytime. In Figure 7, we discussed the allocation of optical slices to each AL. Each optical slice will be allocated to a different application and they, according to their requirements, will request for the VNFs that will be provided to them via the optical domain. Allocating a whole network slice along with required VNFs will give them control on the networking of the slice as well. Considering the per-user/per-application scenario, AL-VC can be modified in such a way that one VC host only one NFC. Each VC and its AL, i.e., optical slice will be working independently providing user with a solicited view of the network. This orchestration will give control to the applications over their VCs. It will make them feel that they are owning the infrastructure. By this, customer satisfaction level can be achieved and that will result in the increased revenue.

\section{VNF Placement}

In the given hybrid domain, due to large bandwidth, optical domain is usually used for large tributary flows. Small flows can be propagated using the electronic domain.

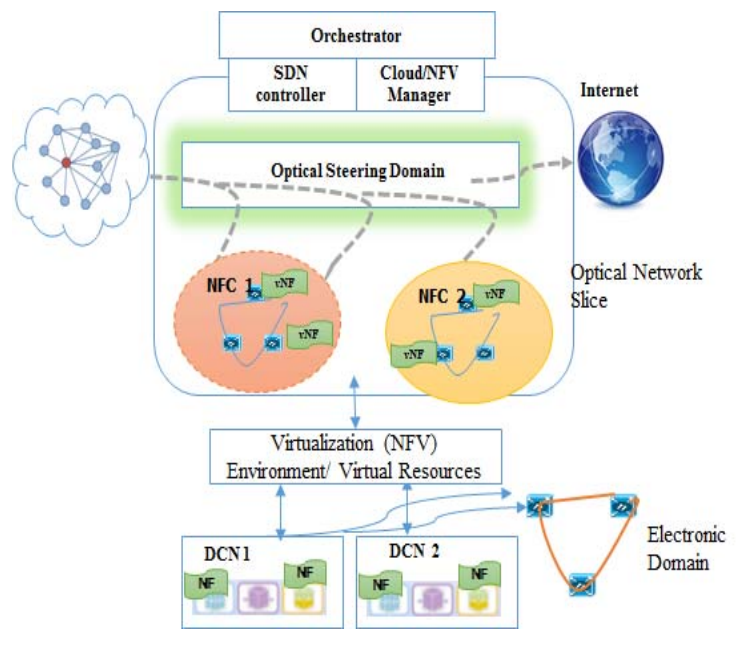

Figure 7. NF/VNFs in the AL-VC 


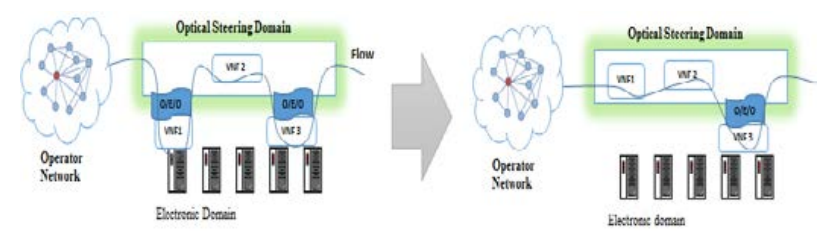

Figure 8. VNF Placement to save O/E/O Conversions

As we mentioned in the previous section that the traffic propagates between electronic and optical domains. When a flow arrives at a DC, it is steered through optical domain, but if a required VNF is on the electronic domain, the flow is converted to electronic traffic and after visiting the VNF, it is converted back to the optical. Each time, the flow is traversed from optical to electronic and back to optical, it consumes $\mathrm{O} / \mathrm{E} / \mathrm{O}$ conversion. Cost of this conversion corresponds to the length of the flow. The larger the flow is, higher will be the cost. Therefore, in order to avoid flow traversing back and forth, we propose to move VNFs to the optical domain as shown in Figure 8, where we considered an NFC consisting of three VNFs. Initially, two VNFs are hosted by the electronic domain; therefore, the flow needs to traverse twice between the optical and electronic domain and consuming two $\mathrm{O} / \mathrm{E} / \mathrm{O}$ conversions. However, by moving one more VNF in the optical domain, we can save another $\mathrm{O} / \mathrm{E} / \mathrm{O}$ conversion.

However, in order to host a VNF, optical routers need to have the storage and processing capabilities. Most of the existing optical routers do not have these capabilities. Therefore, in this work we use the optoelectronic routers. Optoelectronic routers are a special kind of optical routers that have a limited buffer, storage, and processing capability. Therefore, they are capable to host VNFs. Note that, some VNFs' resource demand, e.g., CPU is quite large and that cannot be met by optoelectronic routers. Such VNFs need to be deployed in the electronic domain. Since the optoelectronic routers have limited capabilities, therefore, VNFs only with low resource demands need to be implemented in this domain as in Figure 8, we deployed only two VNFs in the optical domain.

\section{CONCLUSIONS}

In this paper, we discussed our distributed virtual data center network architecture named, AL-VC. Abstraction layer is the key concept of AL-VC. In this work, we presented an efficient algorithm that selects the minimum set of switches to construct the abstraction layers. An abstraction layer along with a group of VMs forms a cluster. This architecture has several advantages and it is very useful in many scenarios. Orchestration of NFCs in NFV environment is one of the main use case of AL-VC. Therefore, using NFV/SDN, we orchestrated multiple NFCs over AL-VC, where one cluster corresponds to one NFC. An NFC consists of several VNFs that can be hosted by either optical domain or electronic domain. However, we showed that moving VNFs in the optical domain can save $\mathrm{O} / \mathrm{E} / \mathrm{O}$ conversions.

\section{ACKNOWLEDGEMENT}

This work was supported by the National Institute of Information and Communications Technology (NICT), Japan.

\section{REFERENCES}

[1] J. S. Turner and D. E. Taylor, "Diversifying the Internet," Proc. Global Telecommunication Conference (GLOBECOM 05) IEEE, Dec. 2005, doi: 10.1109/GLOCOM.2005.1577741.

[2] “Amazon web services: Overview of security rocesses," Amazon, pp. 1-75, Feb. 2015, Available from: https://d0.awsstatic.com/whitepapers/aws-security-whitepaper.pdf , accessed at 2015.12.03

[3] B. Calder, J. Wang, A. Ogus, N. Nilakantan, A. Skjolsvold, and S. McKelvie, "Windows Azure storage: A Highly Available Cloud Storage Service with Strong Consistency," Proc. ACM. 23rd Symposium on Operating System Principles (SOSP 11), 2011, pp. 143-157, doi: 10.1145/2043556.2043571.

[4] N. Farrington and A. Andreyev, "Facebook's Data center Network Architecture," Facebook, Inc. Available from: http://nathanfarrington.com/papers/facebook-oic13.pdf, 2015.12.03

[5] Y. Chen, S. Jain, V. K. Adhikari, Z.L. Zhang, and K. Xu. "A First look at Inter-Data Center Traffic Characteristics via Yahoo! Datasets," Proce. INFOCOM 2011, Apr. 2011, pp. 1620-1628, doi: 10.1109/INFCOM.2011.5934955.

[6] N. M. M. K. Chowdhury, "Network Virtualization: State of the art and Research Challenges," Comm. Mag., IEEE, vol. 47, no. 7, Jul. 2009, pp. 20-26, doi: 10.1109/MCOM.2009.5183468.

[7] T. Anderson, L. Peterson, S. Shenker, and J. Turner, "Overcoming the Internet Impasse through Virtualization,” Comp. IEEE, vol. 38, no. 4, Apr. 2005, pp. 34-41, doi: 10.1109/MC.2005.136.

[8] J. Carapina and J. Jiménez, "Network Virtualization: a view from the bottom," Proc. Workshop on Virtualized Infra Syst. and Arch (VISA 09) ACM, 2009, pp. 73-80, doi: 10.1145/1592648.1592660.

[9] P. Endo, A. Palhares, N. Pereira, G. Goncalves, D. Sadok, and J. Kelner, "Resource Allocation for Distributed Cloud: Concepts and Research Challenges," Netw. IEEE, vol. 25, no. 4, Jul. 2011, pp. 42 46, doi: 10.1109/MNET.2011.5958007.

[10] N. Feamster, L. Gao, and J. Rexford, "How to lease the internet in your spare time," Comp. Comm. Rev. ACM SIGCOMM, vol. 37, 2007, pp. 61-64, doi:10.1145/1198255.1198265.

[11] N. McKeown, T. Anderson, H. Balakrishnan, G. Parulkar, L. Peterson, and J. Rexford, "Openflow: Enabling Innovation in Campus Networks," Comp. Comm. Rev. ACM SIGCOMM, vol. 38, no. 2, pp. 69-74, Apr. 2008, doi: 10.1145/1355734.1355746.

[12] ETSI NFV ISG, "Network Functions Virtualization, An Introduction, Benefits, Enablers, Challenges \& Call for Action," White Paper, http://portal.etsi.org/NFV/NFV_White_Paper.pdf, Oct. 2012.

[13] A.K. Bashir, Y. Ohsita, and M. Murata, "Virtual Clusters for Dynamic Network Service Chaining", Proc. Information and Communictaion Technology and Digital Business Convergence (ICIDB 2015), Nov, 2015, pp. 235-239.

[14] A.K. Bashir, Y. Ohsita, and M. Murata, "Abstraction Layer Based Virtual Clusters Providing Low Network Update Costs in Virtualized Data Centers", Advances in Software., vol. 8, Dec. 2015, pp.457-466, pp.457-466.

[15] A.K. Bashir, Y. Ohsita, and M. Murata, “ Abstraction Layer based distributed architecture for virtualized data centers," Proc. Cloud Computing, GRIDS, and Virtualization (Cloud Computing 2016), Mar. 2015, pp. 46-51.

[16] K. Pentikousis, Y. Wang, and W. Hu, "MobileFlow: Toward Software-Defined Mobile Networks," Communications Magazine, IEEE, vol. 51, no. 7, July 2013, pp. 44-53, doi: 10.1109/MCOM.2013.6553677.

[17] A. Edwards, F. A, and A. Lain, "Diverter: A New Approach to Networking within Virtualized Infrastructures," Proc. Workshop on 
Research on Enterprise Networking (WREN 09) ACM, Aug. 2009, pp. 103-110, doi: 10.1145/1592681.1592698

[18] A. Greenberg, J. Hamilton, N. Jain, S. Kandula, C. Kim, and P. Lahiri, "VL2: A Scalable and Flexible Data Center Network," Proc. Data Communication Conference (SIGCOMM) ACM, Oct. 2009, pp. 51-62, doi: 10.1145/1592568.1592576

[19] T. Benson, A.A.A. Shaikh, and S. Sahu, "CloudNaaS: A Cloud Networking Platform for Enterprise Applications," Proc. Symposium on Cloud Computing (SOCC 11) ACM, Oct. 2011, doi: $10.1145 / 2038916.2038924$.

[20] J. Mudigonda, P. Yalagandula, J. Mogul, B. Stiekes, and Y. Pouffary, "NetLord: A Scalable Multi-Tenant Network Architecture for Virtualized Datacenters," Proc. SIGCOMM, ACM, Aug. 2011, pp. 62-73, doi:10.1145/2018436.2018444.

[21] M. Chowdhury, F. Samuel, and R. Boutaba, "PolyViNE: Policybased Virtual Network Embedding across Multiple Domains," Proc.e Workshop on Virtualized Infrastructure Systems and Architectures (VISA 10) ACM SIGCOMM, Sep. 2010, pp. 49-56, doi: $10.1145 / 1851399.1851408$.

[22] I. Houidi, W. Louati, D. Zeghlache, P. Papadimitriou, and L. Mathy, "Adaptive Virtual Network Provisioning," Proc. 2nd Workshop on Virtualized Infrastructure Systems and Architectures (VISA 10) ACM SIGCOMM, Sep. 2010, pp. 41-48, doi: 10.1145/1851399.1851407.

[23] A.K. Bashir, Y. Ohsita, and M. Murata, "A Distributed Virtual Data Center Network Architecture for the Future Internet," Technical Reports of IEICE (IN2014-165), Mar. 2015, pp. 261-266.

[24] C. Guo, G. Lu, J. H. Wang, S. Yang, C. Kong, and Y.Zhang. "SecondNet: A Data Center Network Virtualization Architecture with Bandwidth Guarantees," in Proceedings of the 6th International Conference (Co-NEXT 10), ACM, 2010, ISBN:978-1-4503-04481 doi: $10.1145 / 1921168.1921188$.

[25] B. Han, V. Gopalakrishnan, L. Ji and S. Lee, "Network Functions Virtualization: Challenges and Opportunities for Innovations," Communications Magazine, IEEE, vol. 53, Feb, 2015, pp. 90-97, doi: 10.1109/MCOM.2015.7045396.

[26] R. Munoz, R. Vilalta, R.Casellas, R.Martinez, T. Szyrkowiec, A. Autenrieth, V. Lopez, D. Lopez, "SDN/NFV orchestration for dynamic deployment of virtual SDN controllers as VNF for multitenant optical networks". Optical Fiber Communication, Conference, Los Angeles, March, 2015.

[27] V. Sekar et al., "The Middlebox Manifesto: Enabling Innovation in Middlebox Deployment," Proc. 10th ACM Workshop. Hot Topics in Networks, 2011.

[28] J. Sherry et al., "Making Middleboxes Someone Else's Problem: Network Processing as a Cloud Service," Proc. ACM SIGCOMM, 2012, pp. 13-24.

[29] Y. Ohsita and M. Murata, "Data Center Network Topologies using Optical Packet Switches," Proc.32nd International Conference on Distributed Computing Systems Workshops (ICDCSW) IEEE, Jun. 2012, pp. 57-64, doi: 10.1109/ICDCSW.2012.53 